\title{
Article pédagogique
}

\section{Immunité et cancers des voies aéro-digestives supérieures $2^{\mathrm{e}}$ partie. Immunothérapies des cancers des VADS}

\author{
Géraldine Lescaille ${ }^{1,2,3}$, Juliette Rochefort ${ }^{1,2,3}$, Rodney Macedo ${ }^{1}$, Aline Le Moignic ${ }^{1}$, \\ Claude Baillou $^{1}$, Véronique Mateo ${ }^{1}$, Chloé Bertolus ${ }^{1,4}$, François M. Lemoine ${ }^{1,5}$
}

1 Sorbonne Universités, UPMC Univ Paris 06, UMR S CR7, INSERM UMR S 1135, Centre d'immunologie et maladies infectieuses (CIMI-Paris), Équipe 8 Immuno-intervention et biothérapies, 75005 Paris, France

2 Département d'odontologie, Hôpital universitaire de la Pitié Salpêtrière, Paris, France

3 Université Paris Diderot, Paris, France

4 Département de chirurgie maxillo-faciale, Hôpital universitaire de la Pitié Salpêtrière, Paris, France

5 Service de biothérapies et Centre d'investigation clinique en biothérapies (CIC-BT 1001), Hôpital universitaire de la Pitié Salpêtrière, Paris, France

(Reçu le 23 décembre 2013, accepté le 27 avril 2014)

Mots clés : cancer des VADS / immunothérapie / vaccination

Key words: head and neck cancer / immunotherapy / vaccination
Résumé - Les cellules immunitaires engendrent des réponses anti-tumorales en reconnaissant les antigènes spécifiques des tumeurs. Un échec du contrôle immunitaire est le plus souvent observé. Ceci est à l'origine de travaux de recherche dont l'objectif est de concevoir l'immunothérapie des cancers. Le meilleur exemple est le développement des anticorps monoclonaux (thérapie ciblée), qui sont une avancée majeure dans le traitement des cancers. Le traitement des cancers des VADS (Voies Aéro-Digestives Supérieures) reste conventionnel, utilisant chirurgie, radiothérapie et chimiothérapie classique. L'objectif de ce travail est une mise au point sur les espoirs de l'immunothérapie dans le traitement des cancers des VADS.

\section{Introduction}

L'immunothérapie consiste à administrer des substances destinées, soit à stimuler les défenses immunitaires de l'organisme pour lutter contre des maladies (cancer ou infections chroniques), soit à moduler des réponses immunitaires mal contrôlées (maladies inflammatoires chroniques et autoimmunes).

La vaccination est un procédé d'immunothérapie consistant à introduire une substance immunogène (l'agent vaccinal) dans un organisme vivant immunocompétent afin de créer une réaction immunitaire spécifique. Les vaccins sont utilisés pour prévenir des pathologies infectieuses. Ces vaccins sont composés d'une molécule naturelle ou recombinante dérivée du pathogène, ou du pathogène lui-même sous une forme inactivée ou atténuée. Les antigènes (substance active du vaccin) stimulent le système immunitaire pour générer une réponse mémoire sans induire de maladie.

La prévention de pathologies infectieuses peut aussi prévenir certains cancers. Certains cancers sont connus pour être

*Correspondance : geraldine.lescaille@gmail.com 
Tableau I. Stratégies vaccinales anti-tumorales.

Table I. Anti-tumoral vaccinal strategies.

\begin{tabular}{|l|}
\hline Immunothérapie « passive » : \\
\hline Anticorps monoclonaux (mAb) dirigés contre les antigènes associés aux tumeurs \\
\hline Administration de cytokines et/ou de chimiokines \\
\hline Ciblage de molécules tolérogènes \\
\hline Transfert adoptif de lymphocytes T \\
\hline Immunothérapie « active » : \\
\hline Vaccination moléculaire : protéines/peptides, pseudo-particules virales (VLP) \\
\hline Vaccination cellulaire ex vivo avec des cellules dendritiques \\
\hline Vaccination génétique : ADN, ARN, vecteurs viraux/non viraux \\
\hline
\end{tabular}

induits par des virus. En neutralisant les agents viraux, les cancers induits par ces virus pourront être prévenus. Différents vaccins sont actuellement disponibles pour empêcher l'infection par les papillomavirus humains (HPV) à haut risque oncogène et donc prévenir les cancers du col de l'utérus, ou encore protéger contre l'hépatite B permettant ainsi d'éviter les hépato-carcinomes induits par ce virus. Toutefois ces vaccins préventifs qui induisent une bonne réponse humorale et des anticorps neutralisants se sont révélés inefficaces dans des lésions cancéreuses. Les stratégies préventives sont à distinguer des vaccinations thérapeutiques anti-tumorales destinées à stimuler ou à renforcer les défenses immunitaires de l'organisme ainsi qu'à diminuer les mécanismes d'échappement des cellules tumorales au système immunitaire. L'immunothérapie est classiquement décrite comme passive ou active selon qu'elle induit ou non une mémoire immunitaire (Tab. I). L'objectif de ce travail est une mise au point sur les espoirs de l'immunothérapie dans le traitement des cancers des VADS.

\section{Immunothérapie passive}

\section{Cytokines}

Les cytokines sont des molécules utilisées dans le traitement de certains cancers qui visent à stimuler le système immunitaire. En effet, la tumeur peut échapper au contrôle du système immunitaire par différents mécanismes, notamment la mise en place d'un micro-environnement tolérogène immunosuppresseur. L'utilisation de certaines cytokines vise à restaurer la fonction effectrice de la réponse immune. Ainsi, on utilise en oncologie l'interleukine 2 (Proleukin ${ }^{\circledR}$ ), ou encore l'IFN alpha (Roreferon- $A^{\circledR}$ ), qui ont fait l'objet d'essais cliniques randomisés. L'IL-2 a pendant plusieurs années été utilisée pour stimuler notamment les lymphocytes T et les NK dans le mélanome et les cancers du rein. Des taux de réponses cliniques de 10 à $15 \%$ ont pu être rapportés [1]. Toutefois, il a été également mis en évidence que l'IL-2 permet de stimuler les lymphocytes T régulateurs, entraînant une potentielle diminution de la réponse effectrice. Ces traitements par cytokines sont associés en combinaison avec des stratégies conventionnelles. Dans les cancers des VADS, aucune étude clinique n'a évalué leur intérêt.

\section{Anticorps monoclonaux}

Les anticorps monoclonaux sont une avancée thérapeutique majeure dans le traitement des cancers. Leur découverte fait suite aux travaux de Georges Köhler et César Milstein durant les années 1970, à partir du constat qu'un cancer des cellules $B$ (myélome) produisait de grandes quantités d'anticorps (Ac) identiques. Par la suite, Greg Winter en 1988 a établi les premières techniques d'humanisation des anticorps monoclonaux ouvrant la porte à une meilleure utilisation médicale chez l'homme en réduisant le risque d'immunogénicité de ces anticorps.

L'utilisation des anticorps monoclonaux est le plus souvent conditionnée par l'expression par les cellules tumorales de l'antigène associé aux tumeurs (TAA). Les anticorps monoclonaux visent ainsi à entraîner la destruction directe des cellules tumorales ou à diminuer la progression tumorale en agissant sur l'angiogenèse tumorale.

Les mécanismes d'action des anticorps monoclonaux sont multiples et complexes :

- blocage d'un facteur de croissance ou de son récepteur indispensable à la prolifération et/ou la survie des cellules tumorales aboutissant à la mort cellulaire le plus souvent par apoptose ;

- cytotoxicité dépendante du complément (CDC). Certains anticorps peuvent activer des protéines du complément. Il en résulte la formation d'un complexe d'attaque membranaire entraînant la lyse cellulaire ;

- cytotoxicité cellulaire dépendante des anticorps (ADCC) : certaines cellules immunitaires comme les monocytes, les macrophages et surtout les cellules NK expriment le récepteur Fc pour les immunoglobulines. Ainsi, la fixation de l'anticorps sur le récepteur Fc de ces cellules entraîne l'activation 
des cellules effectrices et la libération de molécules cytotoxiques (perforines, granzymes) provoquant la lyse des cellules tumorales reconnues par l'anticorps via son site antigénique.

Initialement utilisés dans le traitement des leucémies et des lymphomes, ils occupent aujourd'hui une place de plus en plus importante dans le traitement des tumeurs solides, surtout en association avec les chimiothérapies usuelles. L'association anticorps monoclonaux plus chimiothérapie permet d'améliorer les taux de réponse, d'allonger l'intervalle libre avant la reprise de la progression de la maladie et, dans certaines situations, de prolonger la survie par rapport aux monothérapies respectives.

L'introduction du trastuzumab (Herceptin ${ }^{\circledR}$ ), dans le traitement des carcinomes mammaires HER-2/neu positifs, est une avancée majeure dans le traitement du cancer du sein. Depuis 2011, l'ipilimumab (Yervoy ${ }^{\circledR}$ ), un anti-CTLA4 est d'intérêt majeur dans le traitement du mélanome métastatique [2]. D'autres anticorps tels que le cétuximab (Erbitux ${ }^{\circledR}$, anti-EGFR) et le bévacizumab (Avastin ${ }^{\circledR}$, anti-VEGF) ont permis une nette amélioration de la prise en charge de différents cancers.

Dans les cancers des VADS, certains anticorps monoclonaux sont utilisés. La majorité des études concernent le cétuximab. Il existe une expression d'EGFR dans une grande partie des cancers des VADS et une amplification du gène de l'EGFR dans plus de $30 \%$ de ces cancers [3]. Le cétuximab est utilisé en association à la radiothérapie dans le traitement des cancers des VADS en stade avancé non métastatique [4], ou en combinaison avec la chimiothérapie, notamment dans des stades avancés. Son indication dans ces cancers est encore restreinte [5]. Il a été montré que la combinaison de la radiothérapie et du cétuximab entraînait une forte potentialisation des toxicités. Il existe d'autres types de thérapies ciblées comme les inhibiteurs de la tyrosine kinase dont certains visent le récepteur du facteur de croissance épidermique (EGFR). La plupart n'ont pas fait la preuve d'une efficacité significative, mais le lapatinib (Tyberb ${ }^{\circledR}$ ) et l'afatinib (Giotrif $\left.{ }^{\circledR}\right)$ sont en cours d'évaluation.

Le bévacizumab, approuvé en combinaison avec d'autres thérapies dans différents types de tumeurs solides, est également testé dans les cancers des VADS. Ainsi, une étude de phase III en cours étudie l'intérêt du bévacizumab en association avec d'autres chimiothérapies, dans les cancers récidivants ou métastatiques (NCT00588770). Quelques études de phase II ont rapporté des résultats encourageants avec toutefois des risques sérieux d'hémorragie [6]. Une étude récente montre l'intérêt d'utiliser le bévacizumab en association au cisplatine et à la radiothérapie sans augmenter les toxicités [7].

\section{Transfert cellulaire adoptif}

L'immunothérapie « adoptive » est basée sur le transfert de cellules fonctionnelles, cultivées ex vivo, pour entraîner une réaction cytotoxique contre la tumeur. Ces stratégies cellulaires adoptives, concernent différentes populations cellulaires notamment les lymphocytes T et les cellules Natural Killer (NK). Plus récemment ces stratégies de transfert adoptif ont été renforcées en utilisant des lymphocytes T génétiquement modifiés afin qu'ils expriment des néo-récepteurs antigéniques chimériques (chimeric antigen receptor, CAR) composés à la fois d'une structure d'immunoglobuline et de signaux d'activation lymphocytaires. Des lymphocytes T CAR reconnaissant les molécules CD19 ou CD20 ont été utilisés pour traiter des leucémies aiguës et certains lymphomes avec des résultats extrêmement prometteurs [8]. Peu d'études ont été réalisées dans les cancers des VADS avec ces stratégies.

\section{Immunothérapie active : les vaccins thérapeutiques}

L'immunothérapie active consiste à stimuler les réponses immunitaires adaptatives principalement $T$ de façon à générer des lymphocytes T cytotoxiques (CTL) dirigés contre des antigènes tumoraux et à induire une mémoire immunitaire durable. Pour réaliser ces objectifs, il est donc nécessaire :

- soit d'utiliser des cellules présentatrices d'antigène : des cellules dendritiques qu'on charge ex vivo avec différents antigènes tumoraux. Les cellules sont ensuite réinjectées aux patients : on parle d'immunothérapie cellulaire ;

- soit d'injecter directement ces antigènes tumoraux seuls ou associés à différents vecteurs: ces stratégies moléculaires sont appelées vaccins thérapeutiques.

Pour développer une stratégie vaccinale anti-tumorale, il convient d'identifier un antigène associé à la tumeur. Dans les cancers des VADS, peu d'antigènes ont été étudiés pour la mise au point d'une stratégie vaccinale. Du fait de la présence d'antigènes viraux spécifiques, les cancers viro-induits représentent des cibles de choix pour développer des stratégies d'immunothérapie. La plupart des études dans les cancers des VADS portent sur le développement de stratégie d'immunothérapie dans les cancers induits par le papillomavirus qui représentent 20 à $93 \%$ des cancers de l'oropharynx (amygdale et base de langue). Ceux-ci sont liés aux oncoprotéines E6 et E7 du papillomavirus de type 16 (HPV-16), comme c'est le cas dans la plupart des cancers ano-génitaux. Ces oncoprotéines participent au processus de carcinogenèse en dégradant respectivement le facteur de transcription p53 et la protéine suppressive de tumeur pRb (RB1). Des vaccins utilisant des pseudo-particules virales issues des protéines L1 de la capside de l'HPV ont été développés contre les infections par l'HPV dans le cadre de la prévention des cancers cervicaux. Ces stratégies, qui favorisent la génération d'anticorps neutralisants dirigés contre les protéines structurales de la capside, se sont révélées inefficaces dans le traitement de tumeurs établies, où ce sont les protéines virales non structurales et notamment les antigènes E6/E7 qui 
s'expriment. À ce titre, E6 et E7 représentent des cibles de choix dans le développement de stratégies thérapeutiques des cancers induits par les HPV oncogènes.

À la différence des vaccins prophylactiques qui ciblent les protéines de la capside, les vaccins thérapeutiques se sont focalisés sur des antigènes viraux exprimés dans les lésions malignes liées à l'HPV. En effet, non seulement ces protéines sont impliquées dans la transformation tumorale cellulaire, mais elles sont aussi co-exprimées dans les cellules infectées par l'HPV contrairement aux cellules normales. Tous ces vaccins ont pour but d'induire des lymphocytes T CD8 et CD4 dirigés contre des peptides antigéniques HPV, car ce sont ces réponses cellulaires qui permettraient de détruire les cellules tumorales associées à l'HPV.

\section{Immunothérapie cellulaire}

La vaccination cellulaire concerne principalement les cellules dendritiques (DC) qui représentent la meilleure source de cellules présentatrices d'antigènes pour les lymphocytes $T$ permettant ainsi d'initier des réponses T naïves CD4 et CD8 et de stimuler des réponses mémoires. Ces stratégies impliquent de cultiver des cellules des patients ex vivo, de les modifier in vitro pour qu'elles présentent l'antigène de choix, pour enfin être réinjectées in vivo au patient afin d'induire une réponse adaptative contre l'antigène. L'utilisation ex vivo de $D C$ a été testée chez la souris et dans le cadre d'essais cliniques à partir de $D C$ chargées avec des antigènes exogènes provenant de lysats tumoraux, de cellules apoptotiques, d'exosomes, de peptides ou de DC transduites avec des acides nucléiques (ADN ou ARN) codant les antigènes d'intérêt. Ces études ont montré qu'il était ainsi possible de générer des réponses $T$ cytotoxiques spécifiques et dans quelques cas des réponses cliniques significatives. Toutefois ces stratégies lourdes et coûteuses humainement et financièrement ont donné des résultats peu encourageants : leur développement s'est considérablement réduit. On note cependant une récente application dans les cancers de la prostate. En juin 2010, la FDA (Food and Drug Administration aux États-Unis), après trois essais de phase III, a approuvé la commercialisation du sipuleucel-T (Provenge ${ }^{\circledR}$ ), premier vaccin thérapeutique destiné à des patients atteints de cancer de la prostate résistant aux anti-androgènes mais sans métastases viscérales. Dans le cadre de cancers avancés et en récidive induits par HPV16, une étude de phase I à l'aide de leucocytes stimulés in vitro avec des peptides E6 et E7 est actuellement en cours (Institute NC 2012). Cette stratégie est peu développée dans les cancers des VADS.

\section{Vaccinations moléculaires et génétiques}

Outre la vaccination cellulaire, le développement de stratégies vaccinales portant l'antigène d'intérêt sous une forme génétique à l'aide d'acide nucléique (ADN, ARN), ou encore moléculaire, à l'aide de protéine ou de peptide, a considérablement augmenté. Ces approches vaccinales utilisent des vecteurs qui permettent de véhiculer l'antigène afin d'en augmenter l'efficacité. On distingue des vecteurs viraux et bactériens. Des virus ou des bactéries intra-cellulaires vivants, atténués et génétiquement manipulés pour exprimer des antigènes hétérologues, peuvent être utilisés comme vecteurs pour délivrer des immunogènes au système immunitaire. De tels vecteurs vivants ont l'avantage d'être peu coûteux à produire, permettant une distribution à large échelle. D'autres types de vecteurs sont en cours de développement comme les liposomes ou les pseudo-particules virales (VLP pour Virus Like Particles).

De nombreuses stratégies ont été testées en préclinique et ont montré des résultats intéressants dans des modèles de cancers induits par l'HPV. Cependant, les résultats cliniques sont plus mitigés. Des résultats intéressants ont été observés chez $47 \%$ des patientes dans des lésions dysplasiques de la vulve induites par l'HPV, avec des longs peptides E6/E7 chevauchants [9]; ces résultats étaient accompagnés d'une réponse spécifique CD4 et CD8 de type 1 . Toutefois, ces stratégies n'ont pas permis de traiter des lésions tumorales. Dans les cancers des VADS induits par l'HPV, différentes études sont en cours. Une étude porte sur l'intérêt d'un vecteur bactérien (realistic: ADXS11-001; EUDRACT No : 2010-019916-20) et une autre porte sur l'utilisation d'un vaccin ADN délivré par électroporation.

Dans notre laboratoire, nous avons développé une stratégie de vaccination ADN permettant d'induire in vivo des pseudoparticules virales non infectieuses (VLPs) portant l'antigène viral E7 de l'HVP16. L'intérêt de cette stratégie réside dans le fait que ces VLPs sont produites in vivo après l'administration d'un vecteur plasmidique contenant les séquences nécessaires et suffisantes à l'expression des protéines constitutives des VLPs qui sont ainsi excrétées dans l'environnement extracellulaire. Les VLPs ainsi produites in vivo sont immunogènes et il a été montré que ces VLPs présentaient une meilleure immunogénicité que des protéines solubles. Cette stratégie vaccinale présente l'avantage d'une production simple, rapide, peu onéreuse et transposable à grande échelle.

Nous avons montré que notre stratégie était capable d'induire une réponse lymphocytaire T CD8 spécifique d'E7 in vivo chez l'animal et in vitro chez l'homme. Ainsi, chez l'homme, nous avons montré que la capture de VLP E7 par des DC issues de donneurs de CMH (Complexe majeur d'histocompatibilité) de type HLA-A2 (Human leucocyte antigen), puis mises ensuite en présence de lymphocytes $T$ autologues permettait d'induire une réponse T CD8 spécifique d'E7. Nous avons montré en vaccinant des souris préventivement avec cette stratégie E7 que nous pouvions protéger complètement les animaux contre l'apparition de tumeurs après inoculation des animaux vaccinés par une lignée tumorale TC1 exprimant les oncoprotéines E6 et E7 de HPV16. Dans un modèle curatif sur des tumeurs établies TC1, nous avons induit des régressions 


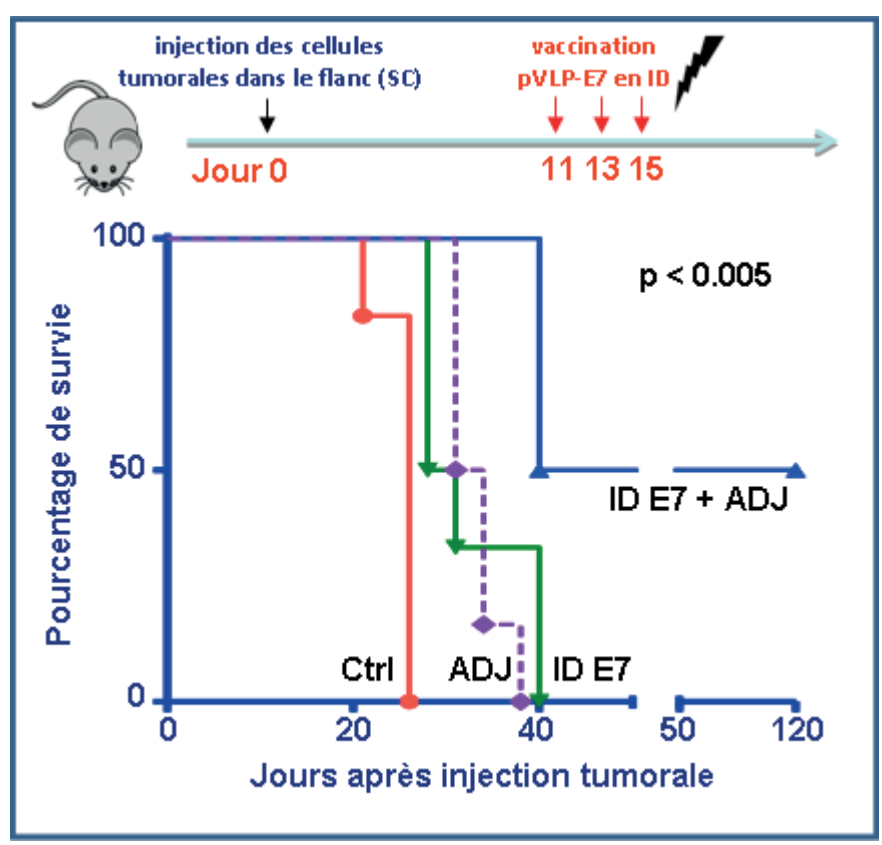

Fig. 1. Réponses anti-tumorales d'une vaccination ADN visant à produire in vivo des pseudo-particules virales (VLP) destinées à induire une réponse CTL dirigée contre l'antigène E7 de l'HPV 16. Des cellules tumorales d'une lignée épithéliale murine exprimant les antigènes de l'HPV 16 (TC-1) ont été injectées en sous-cutané au niveau du flanc (tumeur ectopique). Les souris ont été vaccinées trois fois à deux jours d'intervalle alors que les tumeurs étaient bien établies (jours 11, 13 et 15), soit avec l'ADN E7 seul (ID E7), soit avec les adjuvants seuls (ADJ) (agonistes de TLR 7 et 9) ou en combinaison (ID E7 + ADJ), en comparaison à un groupe contrôle non vacciné (Ctrl). Un avantage statistiquement significatif $(p<0,005)$ à l'utilisation de la combinaison de la vaccination ADN et des adjuvants a été montré pour contrôler la croissance tumorale dans ce modèle.

Fig. 1. Anti-tumoral responses after DNA vaccination producing in vivo virus-like particles (VLPS) designed to elicit a cytotoxic T cell response against E7 antigen from HPV 16. Tumor cells derived from an epithelial tumor cell line expressing HPV 16 E6/ER7 oncoproteins (TC-1 cells) are infused subcutaneously in the flank of mice (ectopic tumors). Then, mice were intradermally vaccinated three times every two days at day 11, 13 and 15 following tumor injection, tumors being well established using either E7 DNA plasmid alone (ID E7), either adjuvants alone (TLR 7 and 9 agonists, $A D J)$, or both (ID E7 + ADJ). Non-vaccinated mice were used as control group (Ctrl). A statistically significant advantage ( $p<0.005)$ of the combination of DNA vaccination + adjuvants to control tumor growth in this model was shown.

statistiquement significatives des tumeurs chez les souris vaccinées à des temps précoces. Nous avons également montré la capacité de cette stratégie à induire des régressions anti-tumorales sur des tumeurs très avancées à condition d'associer les vaccinations avec des adjuvants (Fig. 1) [10]. Ces résultats obtenus à partir d'un modèle antigénique E7 d'HPV16 peuvent être transposés aux antigènes de la famille MAGE notamment MAGE-A4 ou encore NY-ES01 fréquemment retrouvés dans les cancers des VADS non viro-induits $[11,12]$.

\section{Immunothérapies : quelles perspectives ?}

\section{Combinaison à des stratégies conventionnelles}

Depuis quelques années, il a été montré que la chimiothérapie et la radiothérapie pouvaient avoir un effet potentialisateur sur les vaccinations thérapeutiques anti-tumorales. Concernant les chimiothérapies, longtemps considérées comme des traitements antagonistes de l'immunothérapie en raison de leurs effets aplasiants et notamment lymphopéniants, il a été démontré [13] que certaines molécules (anthracyclines, oxiplatine, etc.) pouvaient au contraire participer à l'activité du système immunitaire et contribuer à une meilleure efficacité clinique globale. Cet effet potentialisateur serait lié au recrutement de cellules de l'immunité innée, à la production de cytokines pro-inflammatoires et à la production d'un signal de phagocytose des cellules tumorales par les cellules dendritiques. Certaines chimiothérapies ont une action sur les lymphocytes T régulateurs (Tregs), les cellules NK ou encore les cellules myéloïdes suppressives, ce qui permettrait d'inhiber l'immunosuppression liée au microenvironnement tumoral [14]. Dans les cancers associés à l'HPV, il a été montré que des anticorps anti-DR5 ou l'utilisation du bortézomib (Velcade ${ }^{\circledR}$ ), un inhibiteur du protéasome, permettaient également d'augmenter la réponse de stratégies ADN dans des modèles tumoraux HPV [15]. Ces résultats importants ouvrent la voie à des associations thérapeutiques pertinentes entre certaines 
chimiothérapies et l'immunothérapie. Les stratégies antiangiogéniques semblent être capables d'agir sur le système immunitaire en levant l'immunosuppression. L'administration de sunitinib (Sutent ${ }^{\circledR}$ : antiangiogénique qui inhibe la signalisation en aval du récepteur du Vascular Endothelium Growth Factor, VEGF) chez des patients atteints de cancer du rein, entraîne la diminution des Tregs et des cellules myéloïdes suppressives (MDSC) ainsi que le blocage d'autres mécanismes d'immunosuppression [16]. Ces traitements anti-angiogéniques permettent de potentialiser les stratégies d'immunothérapie dans des modèles précliniques [17]. Ces constatations sont en faveur d'un grand intérêt de l'utilisation combinée de ces stratégies.

La radiothérapie peut stimuler le système immunitaire et présente une action synergique avec des stratégies d'immunothérapie antitumorales ou des immunomodulateurs [18]. Une augmentation de l'expression des molécules de CMH I par la tumeur, la facilitation de la présentation croisée d'antigènes tumoraux par les cellules dendritiques, le recrutement de lymphocytes T intra-tumoraux, la mort immunogénique de la cellule tumorale sont des mécanismes évoqués. Plus récemment, des études précliniques ont étudié l'association des effets de la radiothérapie à faibles doses avec une stratégie ADN antiHPV [19]. Ce traitement permet de rendre les cellules de tumeurs TC-1 plus sensibles à la lyse par des lymphocytes cytotoxiques (CTLs) anti-E7 et d'augmenter significativement les effets curatifs d'une vaccination anti-HPV. Il serait donc intéressant d'associer les effets anti-tumoraux de la radiothérapie à des thérapies immunostimulantes, afin de majorer l'immunité anti-tumorale. La cinétique d'administration du vaccin par rapport à la radiothérapie, le fractionnement (dose par séance) et les doses totales de radiothérapie sont des paramètres importants pour obtenir cette synergie.

\section{Moduler le micro-environnement local}

Les premiers échecs des protocoles d'immunothérapie et une meilleure connaissance du micro-environnement tumoral ont suggéré l'hypothèse selon laquelle l'immunosuppression associée aux tumeurs pouvait expliquer la résistance des tumeurs à l'immunothérapie. Des cellules et des molécules immunosuppressives étaient présentes dans le micro-environnement tumoral.

Certaines cytokines ou certains adjuvants comme des ligands de TLR (récepteur de type toll) ont été décrits comme capables de lever l'état d'anergie des lymphocytes T infiltrant les tumeurs ou de les rendre réfractaires aux mécanismes d'immunosuppression [20]. Différentes molécules peuvent lutter contre l'immunosuppression du micro-environnement tumoral. Il a été proposé d'utiliser des anticorps capables de bloquer la voie PD1/PD1-Ligand (PD1-L). Plusieurs essais cliniques sont actuellement en cours, combinant des anticorps anti-PD1 ou anti-PD1-L avec une stratégie de vaccination (mélanome, cancer de la prostate, carcinome rénal, leucémie aiguë myéloïde), ou avec des anticorps monoclonaux antitumoraux (lymphome), ou avec de la chimiothérapie (cancer du pancréas, cancers du poumon) [21].

Dans les cancers des VADS, cette voie apparaît tout a fait intéressante. Une étude récente montrait une augmentation de l'expression de PD-1 par les LT infiltrant les tumeurs de l'oropharynx associées à l'HPV par comparaison aux autres tumeurs de l'oropharynx, en association à une expression de PDL-1 par plus de $50 \%$ des cellules tumorales. Il a été validé dans un modèle préclinique qu'une tumeur exprimant de façon intrinsèque PDL-1 et n'induisant pas de réponses immunitaires, n'est pas sensible à un anticorps anti-PDL-1. En revanche, la combinaison de cet anticorps avec un vaccin anti-tumoral, qui va provoquer l'induction de LT exprimant PD-1, agit en synergie pour entraîner la régression de la tumeur [22].

\section{Développer des réponses anti-tumorales loco-régionales}

Compte tenu du mauvais pronostic de ces cancers de la muqueuse et du haut risque de récidive loco-régionale, il apparaît important d'avoir de bonnes réponses locales et les voies de vaccination muqueuse constituent une piste intéressante dans les carcinomes épidermoïdes des VADS. Il est bien établi que l'induction d'une immunité muqueuse humorale et cellulaire est mieux corrélée à la protection contre des pathogènes à localisation muqueuse que la seule présence d'une immunité systémique isolée. Des injections muqueuses notamment intranasales et sublinguales permettent non seulement d'obtenir de bonnes réponses immunes systémiques mais aussi muqueuses. Il a été démontré dans de nombreux vaccins anti-infectieux que ces voies de vaccination muqueuses permettaient d'obtenir des réponses anticorps systémiques et muqueuses, localement et à distance. Ces voies permettraient également d'obtenir de bonnes réponses cytotoxiques systémiques mais également muqueuses.

Afin de mieux étudier ces voies muqueuses, il est nécessaire de développer de nouveaux modèles précliniques orthotopiques de cancers des VADS. La plupart des études d'immunothérapie dans ces cancers ont été réalisées sur des modèles de tumeur ectopique sous-cutanée, ou dans des modèles de cancer du col de l'utérus. Nous avons ainsi développé des modèles de tumeurs orthotopiques chez la souris par une injection de cellules de carcinome épidermoïde induit par l'HPV (Fig. 2) dans la langue ou la joue. Nous avons observé que le modèle intrajugal était plus pertinent que le modèle intra-lingual. Le modèle intra-lingual est peu approprié car il comporte un haut risque d'asphyxie chez les souris et rend difficile leur alimentation, aboutissant à une durée de vie réduite. Nous avons donc décidé de choisir le modèle intra-jugal dans l'objectif de tester différentes voies muqueuses d'injection.

Très récemment, Tartour et al. ont montré dans un modèle préclinique de cancer lingual induit par l'HPV que la voie 




Fig. 2. Courbes de survie des souris en fonction du type de modèle ectopique (SC : sous-cutané dans le flanc) ou orthotopique (IJ : intra-jugal ; IL : intra-lingual). Visualisation des tumeurs en bioluminescence dans les 3 modèles tumoraux.

Dans le but de se rapprocher des cancers des VADS induits par l'HPV, nous avons développé des modèles orthotopiques murins à l'aide de la lignée TC1-luciférase. La lignée TC1 constitue la lignée murine épithéliale de référence utilisée dans les modèles précliniques de cancers induits par l'HPV 16. Nous avons testé deux modèles orthotopiques murins en injectant $5.10^{4}$ cellules soit en intra-lingual, soit en intra-jugal. Nous avons ensuite comparé les cinétiques de croissance des différents modèles tumoraux par bioluminescence et par mesure des tumeurs comparativement au modèle classique d'injection dans le flanc des animaux.

Nous avons observé que les souris du modèle intra-lingual présentaient une durée de vie très réduite. Nous avons montré que le modèle intrajugal est le modèle le plus pertinent compte tenu du haut risque d'asphyxie et de difficulté à s'alimenter des souris avec le modèle intralingual. En effet, nous avons observé une perte de poids très importante dans ce modèle en relation avec l'évolution tumorale (non montré). Fig. 2. Mice survival curves according to either ectopic tumor model (SC: subcutaneous injection in the flank) or orthotopic tumor model (IC: intracheek; IL: intra-tongue). Bioluminescence imaging of the three tumor models.

In order to mimic head and neck cancers from the oral cavity, we have set up orthotopic murine models using luciferase-engineered TC-1 cells. TC-1 cells are an epithelial murine cell line usually used for pre-clinical tumor models of HPV 16-induced cancers. Two orthotopic murine models were tested by injecting $5.10^{4}$ cells into either the tongue or the cheek of animals. Tumor growth were monitored by bioluminescence and also measured using a standard method, and compared to a classical tumor model in which tumor cells were injected into the flank of animals.

We first observed a reduced survival life of mice bearing intra-tongue tumors because of breath problems and weight loss related to difficulties to be appropriately fed, this model being not suitable for further experiments. Therefore, the intra-cheek model was kept.

d'administration intra-nasale permettait d'obtenir de meilleures réponses locales que la voie intra-musculaire. Il a également été observé que cette voie intra-nasale permettait d'obtenir localement des lymphocytes CD8+ spécifiques de l'oncoprotéine E7 de HPV 16, exprimant notamment l'intégrine CD49a, un marqueur de homing muqueux des lymphocytes T. Cette induction apparait cruciale, puisque le blocage de CD49a diminue l'infiltrat intra-tumoral des lymphocytes T CD8+ ainsi que la réponse anti-tumorale [23].

La plupart des modèles expérimentaux de cancers induits par l'HPV utilisés dans l'étude de stratégies thérapeutiques relèvent de la greffe sous-cutanée de lignées cellulaires exprimant les antigènes E6 et E7 de l'HVP16. Ces modèles utilisant des lignées cellulaires ont des limites, notamment dans l'étude du micro-environnement tumoral. En effet, l'injection des cellules tumorales permet un développement rapide des tumeurs mais ne permet pas de reproduire les longues interactions entre les cellules immunitaires et tumorales. Différents modèles murins de cancers des VADS ont été développés. Des souris transgéniques pour E6 et E7, K14E6 et K14E7 [24], traitées avec de la 4-nitroquinoline- $\mathrm{N}$-oxide (4-NQ0) développent des cancers de la langue à six mois dans $90 \%$ des cas [25].

\section{Conclusion}

Les cancers des VADS ont encore un pronostic sombre. Le développement des thérapies ciblées et de l'immunothérapie donne de nouveaux espoirs, notamment en combinant les 
stratégies, afin d'individualiser au mieux les traitements en fonction des caractéristiques des tumeurs. Des essais cliniques sont actuellement en cours sur les cancers de l'oropharynx liés à l'HPV. Ce cancer est un modèle en termes d'application thérapeutique dirigée contre un antigène tumoral bien identifié. L'identification d'autres antigènes spécifiques comme ceux de la famille MAGE permettront peut-être à terme de cibler plus spécifiquement des cancers non induits par HPV. Le développement de modèles animaux orthotopiques de cancers des VADS pour tester de nouvelles stratégies thérapeutiques apparaît primordial.

\section{Conflits d'intérêt : aucun}

\section{Références}

1. Negrier S, Escudier B, Lasset C, Douillard JY, Savary J, Chevreau $C$, et al. Recombinant human interleukin-2, recombinant human interferon alfa-2a, or both in metastatic renal-cell carcinoma. Groupe francais d'Immunothérapie. N Engl J Med 1998;338:12721278.

2. Robert C, Thomas L, Bondarenko I, O'Day S, M DJ, Garbe C, et al. Ipilimumab plus dacarbazine for previously untreated metastatic melanoma. N Engl J Med 2011;364:2517-2526.

3. Sheu JJ, Hua CH, Wan L, Lin YJ, Lai MT, Tseng HC, et al. Functional genomic analysis identified epidermal growth factor receptor activation as the most common genetic event in oral squamous cell carcinoma. Cancer Res 2009;69:2568-2576.

4. Bonner JA, Harari PM, Giralt J, Azarnia N, Shin DM, Cohen RB, et al. Radiotherapy plus cetuximab for squamous-cell carcinoma of the head and neck. N Engl J Med 2006;354:567-578.

5. Lefebvre JL, Pointreau Y, Rolland F, Alfonsi M, Baudoux A, Sire $C$, et al. Induction chemotherapy followed by either chemoradiotherapy or bioradiotherapy for larynx preservation: the TREMPLIN randomized phase II study. J Clin Oncol 2013;31:853-859.

6. Cohen EE, Davis DW, Karrison TG, Seiwert TY, Wong SJ, Nattam S, et al. Erlotinib and bevacizumab in patients with recurrent or metastatic squamous-cell carcinoma of the head and neck: a phase I/II study. Lancet Oncol 2009;10:247-57.

7. Fury MG, Lee NY, Sherman E, Lisa D, Kelly K, Lipson B, et al. A phase 2 study of bevacizumab with cisplatin plus intensitymodulated radiation therapy for stage III/IVB head and neck squamous cell cancer. Cancer 2012;118:5008-5014.

8. Ramos CA, Dotti G. Chimeric antigen receptor (CAR)-engineered lymphocytes for cancer therapy. Expert Opin Biol Ther 2011;11:855-73.

9. Kenter GG, Welters MJ, Valentijn AR, Lowik MJ, Berends-van der Meer DM, Vloon AP, et al. Vaccination against HPV-16 oncoproteins for vulvar intraepithelial neoplasia. N Engl J Med 2009, 361:1838-1847.

10. Lescaille G, Pitoiset F, Macedo R, Baillou C, Huret C, Klatzmann D, et al.Efficacy of DNA vaccines forming e7 recombinant retroviral virus-like particles for the treatment of human papillomavirusinduced cancers. Hum Gene Ther 2013;24:533-644.
11. Cesson V, Rivals JP, Escher A, Piotet E, Thielemans K, Posevitz V, et al. MAGE-A3 and MAGE-A4 specific CD4(+) T cells in head and neck cancer patients: detection of naturally acquired responses and identification of new epitopes. Cancer Immunol Immunother 2011;60:23-35.

12. Cuffel C, Rivals JP, Zaugg Y, Salvi S, Seelentag W, Speiser DE, et al. Pattern and clinical significance of cancer-testis gene expression in head and neck squamous cell carcinoma. Int J Cancer 2011;128:2625-2634.

13. Zitvogel L, Apetoh L, Ghiringhelli F, Kroemer G. Immunological aspects of cancer chemotherapy. Nat Rev Immunol 2008;8:59-73.

14. Zitvogel L, Kepp 0 , Kroemer $\mathrm{G}$. Immune parameters affecting the efficacy of chemotherapeutic regimens. Nat Rev Clin Oncol 2011;8:151-60.

15. Tseng CW, Monie A, Wu CY, Huang B, Wang MC, Hung CF, et al. Treatment with proteasome inhibitor bortezomib enhances antigen-specific CD8+ T-cell-mediated antitumor immunity induced by DNA vaccination. J Mol Med (Berl) 2008;86:899-908.

16. Gabrilovich DI, Ishida T, Nadaf S, Ohm JE, Carbone DP. Antibodies to vascular endothelial growth factor enhance the efficacy of cancer immunotherapy by improving endogenous dendritic cell function. Clin Cancer Res 1999;5:2963-2970.

17. Huang KW, Wu HL, Lin $\mathrm{HL}$, Liang PC, Chen PJ, Chen SH, et al. Combining antiangiogenic therapy with immunotherapy exerts better therapeutical effects on large tumors in a woodchuck hepatoma model. Proc Natl Acad Sci U S A 2010;107:1476914774.

18. Lee $Y$, Auh SL, Wang Y, Burnette B, Wang Y, Meng Y, et al. Therapeutic effects of ablative radiation on local tumor require CD8+ T cells: changing strategies for cancer treatment. Blood 2009;114:589-595.

19. Tseng CW, Trimble C, Zeng Q, Monie A, Alvarez RD, Huh WK, et al. Low-dose radiation enhances therapeutic HPV DNA vaccination in tumor-bearing hosts. Cancer Immunol Immunother 2009;58:737-748.

20. Derre L, Rivals JP, Jandus C, Pastor S, Rimoldi D, Romero P, et al. BTLA mediates inhibition of human tumor-specific CD8+ T cells that can be partially reversed by vaccination. J Clin Invest 2010;120:157-167.

21. Topalian SL, Drake CG, Pardoll DM. Targeting the PD-1/B7-H1(PDL1) pathway to activate anti-tumor immunity. Curr Opin Immunol 2012;24:207-212.

22. Badoual C, Hans S, Merillon N, Van Ryswick C, Ravel P, Benhamouda N, et al. PD-1-expressing tumor-infiltrating T cells are a favorable prognostic biomarker in HPV-associated head and neck cancer. Cancer Res 2013;73:128-138.

23. Sandoval F, Terme M, Nizard M, Badoual C, Bureau MF, Freyburger $L$, et al. Mucosal imprinting of vaccine-induced CD8(+) T cells is crucial to inhibit the growth of mucosal tumors. Sci Transl Med 2013; 5:172ra120.

24. Herber R, Liem A, Pitot H, Lambert PF. Squamous epithelial hyperplasia and carcinoma in mice transgenic for the human papillomavirus type 16 E7 oncogene. J Virol 1996;70:1873-1881.

25. Opitz OG, Harada H, Suliman Y, Rhoades B, Sharpless NE, Kent R, et al. A mouse model of human oral-esophageal cancer. J Clin Invest 2002;110:761-769. 